\section{Investigations into mass mortality}

\section{A. Hallam}

Extinction. By Steven M. Stanley. W.H. Freeman: 1987. Pp.242. £15.95.*

A GREAT deal of excitement has been aroused in recent years by theories of extraterrestrially induced mass extinctions, and of extinction periodicity. Hitherto, however, the non-specialist has been frustrated by the lack of a popular but authoritative account of these ideas, putting mass extinctions into the broader context of geological and biological history. Such an account has now been provided by the distinguished palaeontologist Steven Stanley. It comes in the style of an extended article from Scientific American, and as one has come to expect of Stanley the text is lucid, concise and remarkably free of jargon. Quite subtle concepts are explained with refreshing simplicity, and the book is illustrated by a series of excellent colour photographs and diagrams.

Although there have been five major episodes of mass extinction of the world's biota in the past 600 million years, together with a larger number of minor episodes, the greatest recent interest has focused on the end of the Cretaceous partly because of the demise of everyone's favourite creatures, the dinosaurs, and partly because of the much-discussed evidence of an asteroid impact. Accordingly, Stanley devotes most attention to this episode and provides an excellent, well-balanced and remarkably up-to-date description of research on the subject. $\mathrm{He}$ relegates the role of a sudden global catastrophe, resulting from the impact of an extraterrestrial body or spectacular volcanicity, to an insignificant one compared with the longer-term environmental dis-

* In the United States published as a volume in the Scientific American Library.

\section{Published April 1987}

THE STUDY OF CULTURE: REVISED EDITION, by L. L. Langness -288 pages, $6 \times 9^{\prime \prime}$, paper, notes, references, index, illustrated, 1987. $\$ 10.95$, ISBN 0-88316-556-2

Study: Revised is an historically focused introduction to cultural anthropology with an emphasis on the concept of culture that will have strong interdisciplinary appeal. It represents both an expansion and updating of the first edition (1974), which has gone through eight printings and has been widely used in college and university courses

L. L. Langness is Professor of Anthropology and Psychiatry at UCLA.

Order directly from Chandler \& Sharp by sending chec or money order (no credit cards) plus $\$ 1.50$ to cover postage and handling. California customers add appropriate sales tax.

A Chandler and Sharp Publishers, Inc. 11 A Commercial Boulevard Novato. California 94947 turbance produced by a global fall in temperature. Indeed, climatic deterioration as the main promoter of mass extinctions forms the leitmotiv of Stanley's book. In this respect he make a persuasive case for molluscan extinctions in the western Atlantic in the past few million years, and a reasonable one for increased rates of extinction among both marine and terrestrial organisms across the EoceneOligocene boundary.

Further back in time than the late Cretaceous, the evidence supporting his thesis is much more vague and equivocal. Stanley has in consequence to resort in varying degrees to special pleading. Nowhere is this more evident than in the part of the book dealing with the greatest mass extinction episode of all, at the end of the Palaeozoic era. Stanley constantly reiterates the point that tropical organisms such as reef corals have been the most vulnerable to extinction, but such organisms are likely to have exhibited a more general environmental sensitivity than merely to fall in temperature. Even for the apparently well-authenticated case of the Eocene-Oligocene boundary, the issue has been complicated by Shackleton's latest review of oxygen isotope data suggesting that the most severe episode of oceanic temperature fall took place after the extinction of most of the marine organisms in question. While Stanley is right to cast doubt on the widely accepted view that loss of habitat area because of periodic falls of sea-level is a sufficient explanation of marine extinctions, he overstates his case by being selective and partial with the evidence. In particular he relies too uncritically on the sea-level curve of Vail and his associates, ignoring the fact that this curve has been the subject of much dispute. He also underestimates both the sharpness of many episodes of mass extinction as revealed by a close analysis of stratigraphic sections, and the role of anoxic bottom waters spreading episodically across epicontinental seas.

In the present inadequate state of knowledge, it seems that we should keep an open mind about a possible multiplicity of causes, taking due note of the fact that some of them may be intimately linked, such as sea level, climate and volcanicity. There is, after all, no good reason to assume that any two extinction episodes have had precisely the same cause. Perhaps, however, it is better that an author of a book intended for a wide public should enthusiastically promote one particular point of view rather than blandly sit on the fence. Stanley's work certainly makes for stimulating reading.

A. Hallam is Lapworth Professor of Geology, University of Birmingham, PO Box 363, Birmingham B15 2 TT, UK.

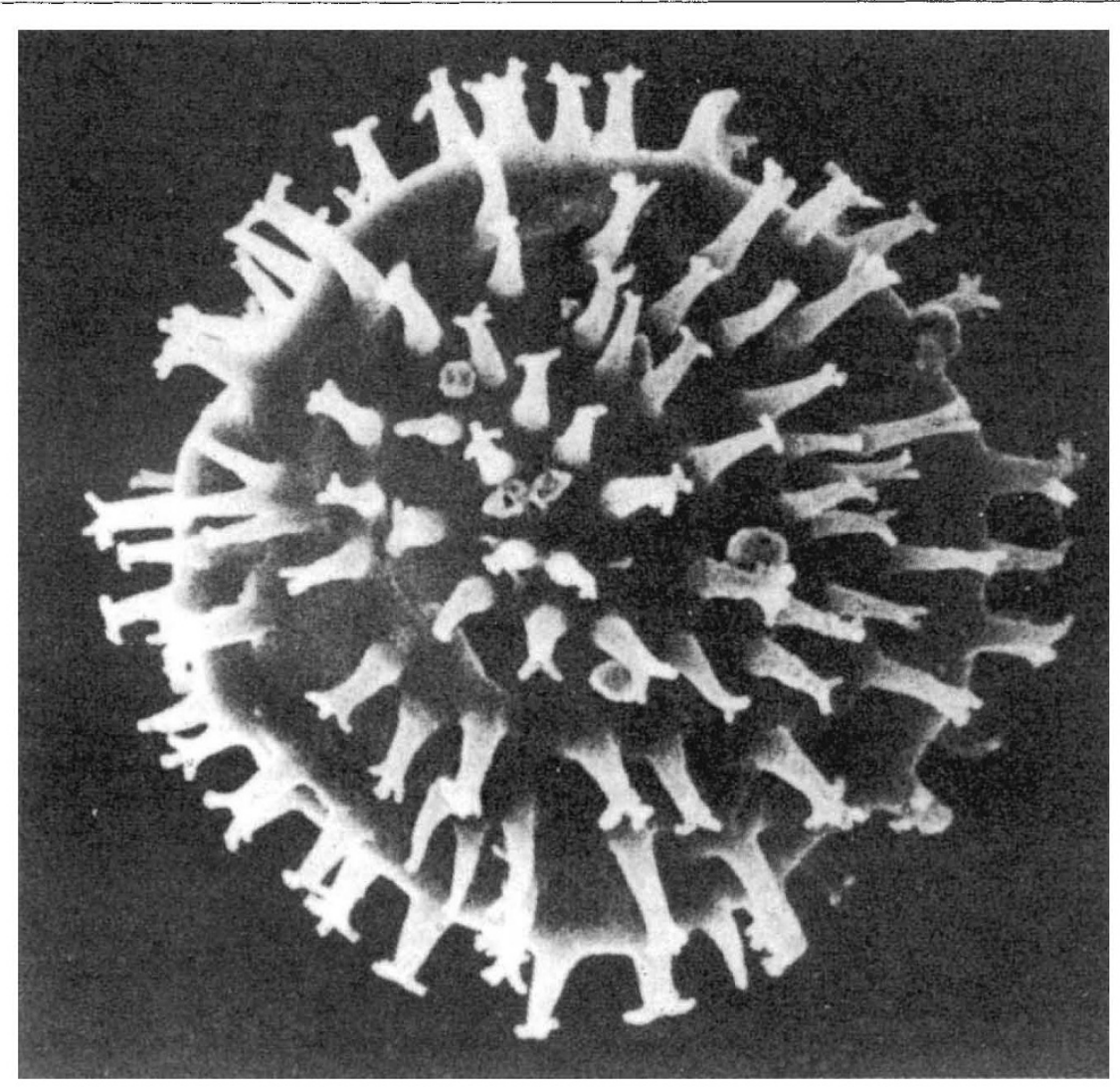

Victim of the first great extinction? An acritarch, the dormant stage of a single-celled alga which is well represented in the fossil record. The diversification of the acritarchs ended suddenly about 650 million years ago, coincidentally with widespread glaciation although the connection remains tentative. 